Pacific Journal of Mathematics

THE MULTIDIMENSIONAL CONTENT OF THE FRUSTUM OF 


\section{THE MULTIDIMENSIONAL CONTENT OF THE FRUSTUM OF THE SIMPLEX}

\section{GIULIO VARSI}

The content of the intersection of a simplex with a semispace is computed by means of a dissection technique. An efficient algorithm, suitable for automatic calculation, is given. For an $n$-dimensional space, the algorithm needs only $n-1$ storage location at most, and requires $\sim n^{2}$ operations.

Introduction. The simplex $S$ considered is the $(n-1)$-dimensional polytope defined as the convex hull of its $n$ vertices $V_{j}$ in $R^{n}$, the Euclidean space in $n$ dimensions:

$$
V_{j} \equiv\left\{v_{j 1}, v_{j 2}, \cdots, v_{j n}\right\},
$$

where

$$
v_{j i}=\delta_{j i}=\left\{\begin{array}{ll}
1 & j=i \\
0 & j \neq i
\end{array} \quad j, i=1,2, \cdots, n .\right.
$$

This choice of geometry is convenient in certain applications of the algorithm that arise in statistical mechanics and allocation theory, but does not result in a loss of generality (see Appendix).

The frustum $F_{1}$ of $S$ is defined as the nonempty intersection of $S$ with the semispace $\sigma$ :

$$
\sigma \equiv\left\{x_{1}, x_{2}, \cdots, x_{n} \mid \sum p_{i} x_{i} \leqq G\right\},
$$

where the real numbers $p_{i}$ are the coefficients that characterize the hyperplane $\gamma$, boundary of the semispace.

With a minor loss of generality, that will be removed subsequently, it will be assumed that

$$
\begin{aligned}
& p_{1}<p_{2}<\cdots<p_{n} . \\
& p_{i} \neq G
\end{aligned} \quad i=1,2, \cdots, n .
$$

It can then be immediately verified that the condition for $S \cap \sigma$ to be nonempty is

$$
p_{1} \leqq G \leqq p_{n} \text {. }
$$

The content $C\left[F_{1}\right]$ of the frustum can be represented as

$$
\iint \cdots \int_{X} d V^{n-1}
$$

where the region $X$ is defined by the following constraints: 


$$
\begin{aligned}
& 0 \leqq x_{i}, i=1,2, \cdots, n, \\
& \sum x_{i}=1, \\
& \sum p_{i} x_{i} \leqq G .
\end{aligned}
$$

The last inequality can also be written as:

$$
\sum m_{i} x_{i} \leqq 0,
$$

where:

$$
m_{i}=p_{i}-G,
$$

and the condition for $S \cap \sigma$ to be nonempty is:

$$
m_{1} \leqq 0 \leqq m_{n} \text {. }
$$

The form $\left(4^{\prime}\right)$ will be used throughout the paper.

It is often convenient to measure $C\left[F_{1}\right]$ in units of $C[S]$, i.e., to express $C\left[F_{1}\right]$ as a fraction of $C[S]$. This latter quantity can be readily evaluated by projecting $S$ onto the $x_{j}=0$ hyperplane and by multiplying the content of the projection by the secant of the angle formed by $S$ with that hyperplane. It results [1]:

$$
C[S]=\frac{\sqrt{n}}{(n-1) !} .
$$

The symbol $\rho^{\prime}$ represents the ratio $C\left[F_{1}\right] / C[S]$.

Properties of the frustum. The hyperplane $\gamma$ defined by

$$
\sum m_{i} x_{i}=0
$$

dissects the simplex $S$ into two frusta $F_{1}$ and $F_{2}$ so that, for any $x \in S$, if $x \in F_{1}$, then $\sum m_{i} x_{i} \leqq 0$; if $x \in F_{2}$, then $\sum m_{i} x_{i} \geqq 0$.

One property of a simplex is that any one of its faces contains all the vertices but one [1]; $\phi_{l}$ denotes that $(n-2)$-face of $S$ which does not contain $V_{l}$, and $E_{i j}$ denotes the edge that connects $V_{i}$ and $V_{j}$. If the hyperplane $\gamma$ cuts $S$, it will partition the vertices $V_{l}$ into two nonempty sets $Z_{1}$ and $Z_{2}$, corresponding to the frusta $F_{1}$ and $F_{2}$. One can distinguish two cases:

(1) Either $Z_{1}$ or $Z_{2}$ contains only one vertex. Let $Z_{1}$ contain only $V_{1}$, then every face $\phi_{l}(l \neq 1)$ will have vertices on both sides of $\gamma$ and, therefore, will be cut by $\gamma ; F_{1}$ will then have a portion of all the $\phi_{l}(l \neq 1)(n-2)$-faces of $S$ plus the $(n-2)$-face lying on $\gamma$, or a total of $n(n-2)$-faces. Furthermore, $F_{1}$ will have as vertices the intersections of all the edges $E_{1 l}(l \neq 1)$ with $\gamma$ plus $V_{1}$ itself. Therefore, $F_{1}$ is a simplex and its content $C\left[F_{1}\right]$ can be evaluated 
immediately by means of the well-known determinant. One also has $C\left[F_{2}\right]=C[S]-C\left[F_{1}\right]$.

(2) Both $Z_{1}$ and $Z_{2}$ contain at least two of the vertices $V_{l}$. Then every face $\phi_{l}$ will have vertices on both sides of $\gamma: \gamma$ cuts every face. Both $F_{1}$ and $F_{2}$ contain a portion (which shall again be called $\phi_{l}$ ) of each face of $S$, plus the common face lying on $\gamma$ (which shall again be called $\gamma$ ) for a total of $n+1(n-2)$-faces. Neither $F_{1}$ nor $F_{2}$ is a simplex. If $Z_{1}$ and $Z_{2}$ contain, respectively, $z_{1}$ and $z_{2}\left(z_{1}+z_{2}=n\right)$ vertices $V_{l}$, then $F_{1}$ contains all the vertices in $Z_{1}$ plus all the intersections of $\gamma$ with the edges $E$ connecting elements of $Z_{1}$ with elements of $Z_{2}$, for a total of $\left(z_{1} \times z_{2}\right)+z_{1}=\left(z_{2}+1\right) z_{1}$ vertices.

LemMa. The frustum $F_{1}$ has the following property: any one of its vertices $W$ belongs to all the $(n-2)$-faces except two.

Proof. If $W$ coincides with one of the $V$, say $V_{l}$, then $W$ belongs to all the $\phi$ except $\phi_{l}$ and does not belong to $\gamma$. If $W$ does not coincide with any $V$ then it belongs to $\gamma$, and it must belong to the edge connecting two of the $V$; let it be $E_{j k}$. Since $V_{j} \in \phi_{h}(h \neq j)$ and $V_{k} \in \phi_{h}(h \neq k)$, then $E_{j k} \in \phi_{h}(h \neq j, k)$ and, since $W \in E_{j k}$, then $W \in \phi_{h}(h \neq j, k)$.

Therefore, $F_{1}$ can be dissected in $\left(z_{2}+1\right) z_{1}$ different ways into two pyramids, ${ }^{1}$ which are characterized by selecting a vertex $W$ and the two faces that do not contain it.

Dissection technique. In this section, the algorithm is described in detail. In essence, the frustum is dissected into two polytopes, which are shown to be pyramids; the bases of these pyramids have the same property as the frustum: namely, each basis can be dissected into two pyramids of one dimension less than the previous ones. The two-fold dissection can be repeated on the new bases until a simplex is obtained. It may appear that the number of two-dimensional pyramids (or triangles) required should be of the order of $2^{n-2}$ and therefore exceed the present computing capabilities for even relatively modest values of $n$. In fact, very substantial simplifications are possible so that the number of operations required is of the order of $n^{2}$.

With no further loss of generality, it will be assumed that

$$
\begin{array}{ll}
m_{i}<0 & i=1, \cdots, J . \\
m_{i}>0 & i=J+1, \cdots, n .
\end{array}
$$

1 A $q$-dimensional pyramid is defined as a polytope with nonnull $q$-dimensional content, such that all its vertices but one belong to a $(q-1)$-flat, called the basis of the pyramid. A $(q-1)$-flat is the set of all points in $(q+k)$ space that can be represented as linear combinations of $q$ linearly independent points $(k \geqq 0)$. 
The hyperplane defined by equation (3) and the faces of $S$ and of $F_{1}$ that belong to it are designated as $\eta$; the hyperplane defined by equation (5) and the faces belonging to it are designated as $\gamma$ as in the previous section. Furthermore, $c_{i}$ indicates the coordinate hyperplane defined by $x_{i}=0$. The intersection of two or more hyperplanes is indicated as a list; $\eta c_{1} c_{7} \gamma$ indicates the $(n-4)$-flat obtained by intersecting the two coordinate hyperplanes $c_{1}$ and $c_{7}$ with $\eta$ and $\gamma$.

From the sign of $\delta=\left\{\sum m_{i} x_{i}\right\}$ it will be noted that all $V_{i}$, for which $1 \leqq i \leqq J$, lie on one side of $\gamma$, and all those $V_{i}$ for which $J<$ $i \leqq n$ lie on the other side [2].

A vertex of $F_{1}$ is indicated as $W_{i}$ if it coincides with the vertex $V_{i}$ of the simplex $S$, or as $W_{l k}$ if it is the intersection of $\gamma$ with the edge $E_{l k}$. (Since $W_{l k} \equiv W_{k l}$, the convention will be followed that $l<$ $k$.) It will be noted that, for any vertex $W_{l k}$, the following inequalities obtain:

$$
1 \leqq l \leqq J<k \leqq n,
$$

because $V_{l}$ and $V_{k}$ have to be on opposite sides of $\gamma$ for $E_{l k}$ to intersect $\gamma$.

The coordinates of $W_{l k}$ can be found immediately by noting that a point $x$ describes the segment $\left[V_{l}, V_{k}\right]$ if

$$
x=\lambda V_{l}+(1-\lambda) V_{k},
$$

where $0 \leqq \lambda \leqq 1$. If $(l, k / i)$ indicates the $i$ th coordinate of $W_{l k}$, then

$$
\begin{aligned}
(l, k / l) & =\left(m_{k}\right) /\left(m_{k}-m_{l}\right), \\
(l, k / k) & =\left(-m_{l}\right) /\left(m_{k}-m_{l}\right), \\
(l, k / i) & =0 \text { for } i \neq l, k,
\end{aligned}
$$

and it is immediately possible to verify that $W_{l k}(=\{(l, k / 1),(l, k / 2)$, $\cdots,(l, k / n)\})$ belongs to $E_{l k}$ and to $\gamma$ :

$$
\sum m_{i}(l, k / i)=m_{l}\left(m_{k}\right) /\left(m_{k}-m_{l}\right)+m_{k}\left(-m_{l}\right) /\left(m_{k}-m_{l}\right)=0 .
$$

The total number of vertices of $F_{1}$ is $N=J+J(n-J)=J(n-J+1)$, as shown in Table I. The $n(n-2)$-faces of simplex $S$ can now be expressed as the intersections of hyperplane $\eta$ with the coordinate hyperplanes: $\eta c_{1}, \eta c_{2}, \cdots, \eta c_{n}$.

THEOREM. The frustum $F_{1}$ can be dissected into two pyramids and, furthermore, the basis of each pyramid can also be dissected into two pyramids; the process can be iterated until the dissection yields simplices.

Proof. The proof will be given by construction and the conditions under which the dissection yields simplices will also be shown. In 
the nontrivial case (2) of the preceding section, $1<J<n-1, F_{1}$ has the appropriate portions of $\eta c_{1}, \eta c_{2}, \cdots, \eta c_{n}$ and the appropriate portion of $\gamma$. It is convenient to dissect $F_{1}$ into two pyramids by selecting the apex on $\gamma$ according to the previous Lemma. Let it be $W_{l_{1} k_{1}}$. Since a vertex $W_{l k}$ belongs to $\eta c_{i}$ if its $i$ th coordinate is zero, then $W_{l_{1} k_{1}}$ belongs to all the faces of $F_{1}$ except $\eta c_{l_{1}}$ and $\eta c_{k_{1}}$. These will be the bases of the two pyramids $\pi^{n-1}\left(l_{1}\right)$ and $\pi^{n-1}\left(k_{1}\right)$; the bases will be designated $\beta^{n-2}\left(l_{1}\right)$ and $\beta^{n-2}\left(k_{1}\right)$. In order to proceed with the dissection, the $(n-3)$-faces of the two bases have to be identified. Consider $\beta^{n-2}\left(l_{1}\right)$. Its faces are the intersections of $\eta c_{l_{1}}$ with the other faces of $F_{1}: \eta c_{l_{1}} c_{l_{2}}$, where $l_{2}=1,2, \cdots, l_{1}-1, l_{1}+1, \cdots, n$. Is the intersection $\eta c_{l_{1}} c_{l_{2}}$ a proper intersection, in the sense that it belongs to $F_{1}$ ? Yes, because $W_{i h} \in \eta c_{l_{1}} c_{l_{2}}$, if $i, h \neq l_{1}, l_{2}$. Analogously, $\eta c_{l_{1}} \gamma$ is a proper intersection, and since $l_{2}$ can take any one of $n-1$ values, then $\beta^{n-2}\left(l_{1}\right)$ has $n-1+1=n(n-3)$-faces. Again, $\beta^{n-2}\left(l_{1}\right)$ has one face more that the simplex of the same $(n-2)$ number of dimensions.

Any one of the vertices of $\beta^{n-2}\left(l_{1}\right)$ can be selected to serve as the apex of the pyramidal dissection, for example, $W_{l_{2} k_{2}}$, where $1 \leqq$ $l_{2} \leqq J<k_{2} \leqq n$ and $l_{2} \neq l_{1}$. (The last condition is necessary because $W_{l_{1} h}(h=J+1, \cdots, n)$ does not belong to $\beta^{n-2}\left(l_{1}\right)$.)

TABLE I. VeRtices of $F_{1}$

\begin{tabular}{|c|c|c|c|c|c|c|c|c|c|}
\hline \multirow{2}{*}{\multicolumn{2}{|c|}{ Vertex }} & \multicolumn{8}{|c|}{ Coordinate } \\
\hline & & 1 & 2 & 3 & $\ldots \ldots$ & $J$ & $J+1$ & $\ldots \ldots$ & $n$ \\
\hline & $W_{1}$ & 1 & 0 & 0 & $\cdots$ & 0 & 0 & $\cdots \cdots$ & 0 \\
\hline & $W_{2}$ & 0 & 1 & 0 & $\ldots$ & 0 & 0 & $\ldots \ldots$ & 0 \\
\hline$J$ & $\cdot$ & $\cdot$ & $\cdot$ & $\cdot$ & $\ldots$. & $\cdot$ & . & $\cdots$ & . \\
\hline & $\cdot$ & $\cdot$ & $\cdot$ & . & $\cdots \cdots$ & $\cdot$ & - & $\ldots \ldots$ & \\
\hline & $W_{J}$ & 0 & 0 & 0 & ..... & 1 & 0 & $\ldots$ & 0 \\
\hline \multirow{6}{*}{$n-J$} & & $m_{, I+1}$ & 0 & 0 & & 0 & $m_{1}$ & & 0 \\
\hline & $W_{1, J+1}$ & $m_{J+1}-m_{1}$ & 0 & 0 & & 0 & $m_{J+1}-m_{1}$ & $\cdots \cdots$ & 0 \\
\hline & . & $\cdot$ & $\cdot$ & • & $\cdots \cdots$ & $\cdot$ & . & $\cdots \cdots$ & . \\
\hline & & . & . & . & $\cdots \cdots$ & . & . & $\cdots \cdots$ & $\cdot$ \\
\hline & $W_{1}$ & $m_{n}$ & 0 & 0 & $\ldots \ldots$ & 0 & 0 & & $-m_{1}$ \\
\hline & $W_{1, n}$ & $m_{n}-m_{1}$ & & & & & & $\cdots$ & $m_{n}-m_{1}$ \\
\hline \multirow{7}{*}{$n-J$} & TT & 0 & $m_{J+1}$ & 0 & & 0 & $-m_{2}$ & & 0 \\
\hline & $W 2, J+1$ & & $m_{J+1}-m_{2}$ & 0 & & 0 & $m_{J+1}-m_{2}$ & $\cdots$ & 0 \\
\hline & . & - & - & . & ..... & - & 0 & .... & - \\
\hline & . & . & . & $\cdot$ & $\cdots \cdots$ & - & 0 & $\cdots \cdots$ & - \\
\hline & $\cdot$ & - & - & . & $\cdots \cdots$ & $\cdot$ & - & $\cdots \cdots$ & $\cdot$ \\
\hline & $W_{J, n}$ & 0 & 0 & 0 & .. & $\frac{m_{n}}{m_{n}-m_{J}}$ & 0 & ..... & $\frac{-m_{J}}{m_{n}-m_{J}}$ \\
\hline & & & & & & & & & $m n-m J$ \\
\hline
\end{tabular}

The two new bases are those faces of $\beta^{n-2}\left(l_{1}\right)$ that do not contain 
$W_{l_{2} k_{2}}$. These faces are $\eta c_{l_{1}} c_{l_{2}}$ and $\eta c_{l_{1} k_{2}}$. The shorter notation $\beta^{n-3}\left(l_{1}, l_{2}\right)$ and $\beta^{n-3}\left(l_{1}, k_{2}\right)$ will be employed.

In general, the $\mu$-dimensional base of a $(\mu+1)$-dimensional pyramid will be indicated by $\beta^{\mu}\left(h_{1}, h_{2}, \cdots, h_{n-\mu-1}\right)$. Its $\mu+2(\mu-1)$-dimensional faces are:

$$
\eta c_{h_{1}} c_{h_{2}} \cdots c_{h_{n-\mu-1}} c_{i}
$$

and

$$
\eta c_{h_{1}} c_{h_{2}} \cdots c_{h_{n-\mu-1}} \gamma
$$

where $i \neq h_{1}, h_{2}, \cdots, h_{n-\mu-1}$.

The argument $h_{1}, \cdots, h_{n-\mu-1}$ is a combination without repetition of $n-\mu-1$ among the $n$ numbers $1,2, \cdots, n$.

The next dissection is accomplished by selecting a vertex $W_{l k}$ with the usual conditions on $l$ and $k$ with the further limitation

$$
l, k \neq h_{i} \quad i=1, \cdots, n-\mu-1 .
$$

The new bases are $\beta^{\mu-1}\left(h_{1}, \cdots, h_{n-\mu-1}, l\right)$ and $\beta^{\mu-1}\left(h_{1}, \cdots, h_{n-\mu-1}, k\right)$.

It is convenient to represent the pyramidal dissection geometrically with a tree. Each node of the tree represents a pyramid and two branches issue from it.

It has been noted that one of the pyramids may in fact be a simplex; therefore, the dissection process ends on that branch. This occurs where one of the intersections of the base of the pyramid with the remaining coordinate hyperplanes or with $\gamma$ is improper. A generic intersection $I \equiv \eta c_{h_{1}} \cdots c_{h_{l}} \cdots c_{h_{\nu}}$ (where $h_{\nu}=0$ indicates $\gamma$ ) is improper if it does not contain any one of the $W_{l k}$ or $W_{i}$. This happens in either of the following two cases:

(1) If for each $i \leqq J$ there exists an $h_{l} \ni h_{l}=i$. Since all the vertices of $F_{1}$ have a nonzero coordinate, the cardinal number $i$ of which is less than or equal to $J$, no vertex can have zero coordinate for all $i \leqq J$, and therefore no vertex can belong to $I$.

(2) If $h_{l}=0$ for some $l$ and for each $i \geqq J+1$ there exists an $h_{l} \ni h_{l}=i$. Since all the vertices that belong to $\gamma$ have a nonzero coordinate the cardinal number $i$ of which is greater than $J+1$, no vertex can belong to $I$.

A base $\beta^{n-\nu-1}$ that forms an improper intersection has only $n-\nu$ $(n-\nu-2)$-faces, rather than $n-\nu+1$ and is, therefore, a simplex. This occurs if the argument list of $\beta^{n-\nu-1}$ contains $J-1$ indices smaller than or equal to $J$ (one of the intersections of $\beta^{n-\nu-1}$ will fall under Case 1) or if it contains all the $n-J$ indices greater than $J$ (Case 2). The conditions stated above can be verified also by considering 
the number of vertices of a base $\beta^{n-\nu-1}\left(h_{1}, h_{2}, \cdots, h_{2}\right)$. Since the order of the arguments in the list is irrelevant, we may assume that they are ordered as follows:

$$
h_{1}<h_{2}<h_{3}<\cdots<h_{q} \leqq J<\cdots<h_{\nu} .
$$

By inspection of Table I one can verify that from the total number $N=J(n-J+1)$ of vertices of $F_{1},(n-J+1)$ are excluded for each of the $h_{i} \leqq J$ and $(J-q)$ are excluded for each one of the $h_{i} \geqq J+1$. The number $N_{\beta}$ of vertices belonging to that base is

$$
\begin{aligned}
N_{\beta} & =J(n-J+1)-(n-J+1) q-(J-q)(\nu-q) \\
& =(J-q)(n+1-J-\nu+q) .
\end{aligned}
$$

Since the base $\beta^{n-\nu-1}$ is of dimension $n-\nu-1$, it is a simplex if it has $N_{\beta}=(n-\nu-1)+1=n-\nu$. This condition, a second-degree equation in $q$, has two solutions: $q_{1}=J-1$ and $q_{2}=J+\nu-n$. The first condition is clearly equivalent to the one enunciated previously that led to case (1); the second can be rewritten as $\nu-q_{2}=n-J$ and can be shown to coincide with the second condition given above. The proof of the statement of the theorem is thus complete.

Now, the distance of the apex of the pyramid from its base has to be evaluated. In general, this is the distance of $W_{l k}$ from $\beta^{\mu}\left(h_{1}\right.$, $\left.h_{2}, \cdots, h_{n-\mu-2}, k\right)$. This quantity has a somewhat cumbersome expression because it is the distance between a point and a $\mu$-flat in $R^{\mu+2}$. (The vertex $W_{l k}$ and the vertices belonging to $\beta^{\mu}$ collectively have $n-(n-\mu-2+1)+1=\mu+2$ coordinates different from zero.) The equivalent case in $R^{3}$ is the determination of the distance of a point from a straight line lying on one of the coordinate planes. The expression, however, becomes very simple if, as previously suggested, $F_{1}$ is projected onto one of the coordinate hyperplanes.

Since the cosines of $\eta$ are all equal, the relationship $C\left[F_{1}\right]=$ $C\left[\Phi_{1}\right](n)^{1 / 2}$ holds for $\Phi_{1}$, the projection of $F_{1}$ on any one of the coordinate hyperplanes. The only condition is that neither $c_{l}$ nor $c_{k}$ be used in connection with $W_{l k}$. As will be shown subsequently, it is quite convenient to use $c_{J}$ as the projection hyperplane for all the pyramids. In the projection plane, the distance between the projections of $W_{l k}$ and $\beta^{\mu}\left(h_{1}, h_{2}, \cdots, n_{n-\mu-2}, k\right)$ is simply the $k$ th coordinate of $W_{l k}:(l, k / k)=$ $\left(-m_{l}\right) /\left(m_{k}-m_{l}\right)$. This statement can be verified by minimizing the square of the distance $d$ between the projections of a generic point $x$ on $\beta^{\mu}$ and $W_{l k}$ as follows:

$$
\begin{aligned}
d^{2} & =\sum\left[x_{i}-(l, k / i)\right]^{2} \\
& =(l, k / k)^{2}+\sum^{*} x_{i}^{2}+\left[x_{l}-(l, k / l)\right]^{2},
\end{aligned}
$$


where $\sum^{*}$ indicates the sum for $i \neq h_{1}, h_{2}, \cdots, h_{n-\mu-2}, k, l, J$, and where the following constraints apply:

$$
\Sigma^{+} x_{i} \leqq 1, \quad 0 \leqq x_{i} \leqq 1 \text { for } i \neq J .
$$

Here, $\Sigma^{+}$indicates the sum for $i \neq J$. It can be verified that $d^{2}$ is minimum when all its terms but the first are zero:

$$
\begin{aligned}
& d^{2}=(l, k / k)^{2}, \\
& x_{i}=0 \text { for } i \neq l, \\
& x_{l}=(l, k / l) .
\end{aligned}
$$

The content of the pyramid of base $\beta^{\mu}$ and apex $W_{l k}$ is then computed from the content of its projection $\beta$ as $[2,3]$ :

$$
\left(n^{1 / 2}\right)[1 /(\mu+1)]|(l, k / k)| C\left[\beta\left(h_{1}, h_{2}, \cdots, h_{n-\mu-2}, k\right)\right] .
$$

Description of the algorithm. The rules for the construction of the tree representing the dissection can be obtained from the rules given in the previous section. These rules are summarized as follows:

(1) Start with node $\Phi_{1}$.

(2) From $\Phi_{1}$ go to the two nodes labeled $l_{1}$ and $k_{1}$, where

$$
1 \leqq l_{1} \leqq J ; \quad J+1 \leqq k_{1} \leqq n
$$

(3) From each node draw two branches to two further nodes and label them $l_{\mu}$ and $k_{\mu}$, where $1 \leqq l_{\mu} \leqq J$ and $J+1 \leqq k_{\mu} \leqq n$ and where both $l_{\mu}$ and $k_{\mu}$ are different from all the labels affixed to the previous nodes in the path leading from $\Phi_{1}$ to the nodes labeled, respectively, $l_{\mu}$ or $k_{\mu}$, and so on.

(4) A path is terminated:

(a) If $J-1$ of its nodes, including the terminal one, have labels of numerical value $\leqq J$ or

(b) if $n-J$ of its nodes, including the terminal one, have labels of numerical value $\geqq J+1$.

The construction can be systematized by the addition of the following rule:

(5) The label assigned to each node is the lowest possible, provided that rule 3 also is observed.

Two symbols are associated with each node: One is the label discussed previously, the other is an element of matrix $A=\left\|a_{r, s}\right\|$, where $r=J-h_{q}$ and $s=n+1-h_{\nu}, h_{q}$ and $h_{\nu}$ being defined as in equation (7).

The value of the matrix element is the content of the projection of the base of the pyramid represented by the node. It should be noted that if two nodes are reached by paths containing the same 
labels, they have the same value; furthermore, the paths exiting from them are identical.

An obvious consequence of rules 4 and 5 is that label $J$ never appears and that the label of a terminal node is either $J-1$ or $n$. The computation starts from the pyramid whose matrix element has the lowest indices (to the end of this section: $(l, k / i) \equiv|(l, k / i)|)$ :

$$
\begin{aligned}
a_{2,2}= & C[\beta(1,2, \cdots, J-3, J-2, J+1, \cdots, n-2, n-1)] \\
= & (1 / 2)\{(J-1, n / J-1) C[\beta(1,2, \cdots, J-2, J+1, \cdots, n-1, J-1)] \\
& +(J-1, n / n) C[\beta(1,2, \cdots, J-2, J+1, \cdots, n-1, n)]\} \\
& =(1 / 2)\left\{(J-1, n / J-1) a_{12}+(J-1, n / n) a_{21}\right\} .
\end{aligned}
$$

Next:

$$
\begin{aligned}
& a_{2,3}=(1 / 3)\left\{(J-1, n-1 / J-1) a_{1,3}+(J-1, n-1 / n-1) a_{2,2}\right\}, \\
& a_{2,4}=(1 / 4)\left\{(J-1, n-2 / J-1) a_{1,4}+(J-1, n-2 / n-2) a_{2,3}\right\}, \text { etc. }
\end{aligned}
$$

The last element evaluated in this phase of the calculation is

$$
\begin{aligned}
a_{2, n-J+1}= & (n-J+1)^{-1}\left\{(J-1, J+1 / J-1) a_{1, n-J+1}\right. \\
& \left.+(J-1, J+1 / J+1) a_{2, n-J}\right\} .
\end{aligned}
$$

The next phase requires the evaluation of

$$
\begin{aligned}
C[\beta(1,2, \cdots, J-3)]= & a_{3, n-J+1} \\
= & (n-J+2)^{-1}\left\{(J-2, J+1 / J-2) a_{2, n-J+1}\right. \\
& \left.+(J-2, J+1 / J+1) a_{3, n-J}\right\} .
\end{aligned}
$$

While the element $a_{2, n-J+1}$ has just been calculated, $a_{3, n-J}$ is determined as follows:

$$
\begin{aligned}
a_{3, n-J} & =C[\beta(1,2, \cdots, J-3, J+1) \\
& =(n-J+1)^{-1}\{(J-2, J+2 / J-2) C[\beta(1,2, \cdots, J-3, J+1, J-2)] \\
& +(J-2, J+2 / J+2) C[\beta(1,2, \cdots, J-3, J+1, J+2)]\} .
\end{aligned}
$$

The time-saving technique derives from the observation that, in the above expression,

$$
\begin{aligned}
& C[\beta(1,2, \cdots, J-3, J+1, J-2)] \\
= & C[\beta(1,2, \cdots, J-3, J-2, J+1)]=a_{2, n-J},
\end{aligned}
$$

which has been computed.

The general term has the form:

$$
\begin{aligned}
a_{h, k}= & \{(h+k-2)\}^{-1}\left\{a_{h, k-1}(J-h+1, n-k+2 / n-k+2)\right. \\
& \left.+a_{h-1, k}(J-h+1, n-k+2 / J-h+1)\right\}
\end{aligned}
$$

for $2 \leqq h \leqq J$ and $2 \leqq k \leqq n-J+1$. 
It can be verified, by evaluation of the appropriate determinant, that the contents of the projections of the simplices represented by the first row and first column of matrix $A$ are:

$$
\begin{aligned}
a_{1, k} & =\{(k-1) !\}^{-1}\left\{\prod_{0}^{k-2}(J, n-i / n-i)\right\} \\
& =\{(k-1) !\}^{-1}\{(J, n / n)(J, n-1 / n-1) \cdots(J, n-k+2 / n-k+2)\},
\end{aligned}
$$

or, recursively,

$$
a_{1, k}=a_{1, k-1}(J, n-k+2 / n-k+2)(k-1)^{-1},
$$

for $2 \leqq k \leqq n-J+1$, and

$$
a_{h, 1}=\{(h-1) !\}^{-1},
$$

for $2 \leqq h \leqq J$, while the element $a_{1,1}$ is indeterminate.

The content of the projection of $F_{1}$ is determined as:

$$
C\left[\Phi_{1}\right]=C\left[F_{1}\right](n)^{-1 / 2}=a_{J, n-J+1},
$$

the matrix element evaluated last.

Numerical implementation of the algorithm. It will be noted that since the calculation of each element $a_{h, k}$ of matrix $A$ requires knowledge only of the value of the elements immediately above and to the left of it, the actual machine calculation needs to store only one row (or one column) of the matrix. Furthermore, since the quantity $\rho^{\prime}$ is expressed as $\rho^{\prime}=C\left[F_{1}\right] / C[S]$, where a factor $n^{1 / 2}\{(n-1) !\}^{-1}$ appears in the numerator and in the denominator, the calculation can be simplified if one avoids the carrying of the divisor. If, for example, one computes and stores by rows, one obtains:

$$
\rho^{\prime} \leftarrow \alpha_{n-J+1}
$$

where a computational rather than mathematical notation has been used and where the result is obtained by the following steps:

(1) $\alpha_{1}=1, \alpha_{2}=\alpha_{3}=\cdots=\alpha_{n-J+1}=0$.

(2) Repeat step 3 for $h=1,2,3, \cdots, J$.

(3) $\alpha_{k} \longleftarrow \alpha_{k-1} \frac{\left|m_{J+1-h}\right|}{\left|m_{J+1-h}-m_{n+2-k}\right|}+\alpha_{k} \frac{\left|m_{n+2-k}\right|}{\left|m_{J+1-h}-m_{n+2-k}\right|}$,

for $k=2,3, \cdots, n-J+1$, where $J$ is defined as in equation (6).

Since $\alpha_{n-J+1}$ as a function of $m_{i}$ is defined and continuous in the region defined by the following inequalities,

$$
m_{1} \leqq m_{2} \leqq \cdots \leqq m_{J} \leqq 0<m_{J+1} \leqq m_{J+2} \leqq \cdots \leqq m_{n}
$$

and since $\rho^{\prime}$ is also continuous because of its geometrical interpretation, the restrictions instituted before can be removed and the region of applicability of the algorithm extended as shown by the above inequalities. 
Except for the first iteration of step 2, the evaluation of the generic $\alpha_{k}$ involves 6 elementary operations ( 1 sum, 2 differences, 2 multiplications, and 1 division). Each iteration of step 3, therefore, requires $6(n-J)$ operations, but the first iteration requires only $4(n-J)$. The complete calculation requires $M$ operations:

$$
M=4(n-J)+[6(n-J)]+1](J-1) .
$$

The maximum value $M_{\max }$ that $M$ can assume for a given $n$ is

$$
M_{\max }=\left(3 n^{2}-n\right) / 2-1 \text {. }
$$

The storage requirement for the vector $\alpha_{i}$ is $n-J+1$ locations.

Appendix. A generic $r$-dimensional frustum $F^{\prime}$ given as the intersection of the semispace $\sigma^{\prime}\left(\sigma^{\prime} \equiv\left\{\xi_{1}, \xi_{2}, \cdots, \xi_{r} \mid \sum_{i=1}^{r} g_{i} \xi_{i} \leqq g_{0}\right\}\right)$ and the simplex $S^{\prime}\left(S^{\prime} \equiv\right.$ convex hull of $P_{i}$, for $i=1,2, \cdots, r+1$, where $\left.P_{i} \equiv\left\{q_{i 1}, q_{i 2}, \cdots, q_{i r}\right\}\right)$ can be reduced to a frustum $F$ of the specialized form assumed herein by means of the following change of variables:

$$
\xi_{i}=\xi_{i}^{0}+\sum_{1}^{r} \tau_{i j} x_{j},
$$

where

$$
\begin{aligned}
\xi_{i}^{0} & =q_{r+1, i}, \\
\tau_{i j} & =q_{j i}-q_{r+1, i} .
\end{aligned}
$$

The semispace $\sigma^{\prime}$ transforms into $\sigma^{\prime}$ as follows:

$$
\begin{aligned}
\sigma & \equiv\left\{x_{1}, x_{2}, \cdots, x_{r} \mid \sum_{1}^{r} g_{i}\left(\xi_{i}^{0}+\sum_{1}^{r} \tau_{j} \tau_{i j} x_{j}\right)\right. \\
& \left.=\sum_{1}^{r} g_{i} \xi_{i} \xi_{i}+\sum_{1}^{r} x_{j}\left(\sum_{1}^{r} \tau_{i} \tau_{i j} g_{i}\right) \leqq g_{0}\right\} .
\end{aligned}
$$

Using the notation established herein, setting $r+1=n$, and using the prime (') to indicate that the sequence of coefficients $p^{\prime}$ has to be ordered before applying the algorithm, we obtain

$$
\begin{aligned}
p_{j}^{\prime} & =\sum_{1}^{r}{ }_{i} g_{i}\left(q_{j i}-q_{r+1, i}\right), \quad j=1,2, \cdots, r \\
G & =g_{0}-\sum_{i}^{r} g_{i} q_{r+1, i}, \\
p_{r+1}^{\prime} & =p_{n}^{\prime}=0 .
\end{aligned}
$$

It can be verified that

$$
C\left[F^{\prime}\right]=C[F]\left\{\operatorname{det}\left\|\tau_{i j}\right\|\right\}=(r !)^{-1} \alpha_{n-J+1} \cdot \operatorname{det}\left\|\tau_{i j}\right\|(r+1)^{1 / 2}
$$

where $\alpha_{n-J+1}$ is computed as shown previously. 


\section{REFERENCES}

1. H. S. M. Coxeter, Regular Polytopes, The MacMillan Co., New York, 1963.

2. D. Gale, On the number of faces of a convex polytope, Canad. J. Math., 16 (1964), 12.

3. D. M. Y. Sommerville, An Introduction to the Geometry of $n$ dimensions, Methuen and Co.. Ltd., London, 1929.

Received January 5, 1971 and in revised form November 16, 1972. This paper presents the results of one phase of research carried out at the Jet Propulsion Laboratory, California Institute of Technology, under Contract No. NAS 7-100, sponsored by the National Aeronautics and Space Administration. The author acknowledges the encouraging conversation with Prof. B. Grunbaum, of the University of Washington, and the long discussion with Dr. F. Krogh, of the Jet Propulsion Laboratory, who suggested a simplification in the algorithm.

National Research Council, Washington, D. C.

Present address: Jet Propulsion laboratory, Pasadena, California 


\section{PACIFIC JOURNAL OF MATHEMATICS}

\section{EDITORS}

D. Gilbarg and J. Milgram Stanford University

Stanford, California 94305

R. A. Beaumont

University of Washington

Seattle, Washington 98105
J. DugundJI

Department of Mathematics

University of Southern California

Los Angeles, California 90007

RICHARD ARENS

University of California

Los Angeles, California 90024

ASSOCIATE EDITORS
E. F. BECKENBACH
B. H. NeumanN
F. WOLF
K. YoSHIDA

\section{SUPPORTING INSTITUTIONS}

UNIVERSITY OF BRITISH COLUMBIA

CALIFORNIA INSTITUTE OF TECHNOLOGY

UNIVERSITY OF CALIFORNIA

MONTANA STATE UNIVERSITY

UNIVERSITY OF NEVADA

NEW MEXICO STATE UNIVERSITY

OREGON STATE UNIVERSITY

UNIVERSITY OF OREGON

OSAKA UNIVERSITY
UNIVERSITY OF SOUTHERN CALIFORNIA

STANFORD UNIVERSITY

UNIVERSITY OF TOKYO

UNIVERSITY OF UTAH

WASHINGTON STATE UNIVERSITY

UNIVERSITY OF WASHINGTON

$\stackrel{*}{*} \stackrel{*}{*} \stackrel{*}{*}$ AMERICAN MATHEMATICAL SOCIETY

NAVAL WEAPONS CENTER 


\section{Pacific Journal of Mathematics}

\section{Vol. 46, No. $1 \quad$ November, 1973}

Allan Francis Abrahamse, Uniform integrability of derivatives on $\sigma$-lattices .......................................... 1

Ronald Alter and K. K. Kubota, The diophantine equation $x^{2}+D=p^{n} \ldots \ldots \quad 11$

Grahame Bennett, Some inclusion theorems for sequence spaces .......... 17

William Cutler, On extending isotopies ........................ 31

Robert Jay Daverman, Factored codimension one cells in Euclidean

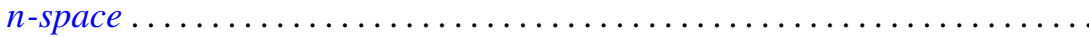

Patrick Barry Eberlein and Barrett O’Neill, Visibility manifolds ............ 45

M. Edelstein, Concerning dentability .......................... 111

Edward Graham Evans, Jr., Krull-Schmidt and cancellation over local

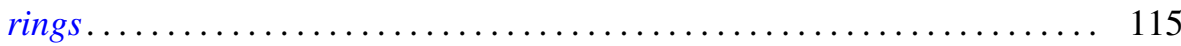

C. D. Feustel, A generalization of Kneser's conjecture ................ 123

Avner Friedman, Uniqueness for the Cauchy problem for degenerate parabolic equations .......................................... 131

David Golber, The cohomological description of a torus action ............ 149

Alain Goullet de Rugy, Un théorème du genre "Andô-Edwards" pour les

Fréchet ordonnés normaux..............................

Louise Hay, The class of recursively enumerable subsets of a recursively enumerable set ........................................

John Paul Helm, Albert Ronald da Silva Meyer and Paul Ruel Young, On orders of translations and enumerations...

Julien O. Hennefeld, A decomposition for $B(X)^{*}$ and unique Hahn-Banach

extensions

Gordon G. Johnson, Moment sequences in Hilbert space .

Thomas Rollin Kramer, A note on countably subparacompact spaces ...

Yves A. Lequain, Differential simplicity and extensions of a derivation ....

Peter Lorimer, A property of the groups Aut $\mathrm{PU}\left(3, q^{2}\right) \ldots$

225

Yasou Matsugu, The Levi problem for a product manifold.

231

John M.F. O'Connell, Real parts of uniform algebras ......

William Lindall Paschke, A factorable Banach algebra without bounded

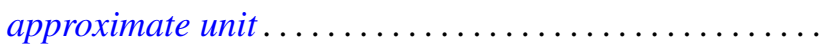

Ronald Joel Rudman, On the fundamental unit of a purely cubic field ....

Tsuan Wu Ting, Torsional rigidities in the elastic-plastic torsion of simply connected cylindrical bars .........................

Philip C. Tonne, Matrix representations for linear transformations on analytic sequences...................................

Jung-Hsien Tsai, On E-compact spaces and generalizations of perfect

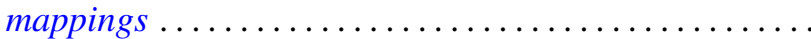

Alfons Van Daele, The upper envelope of invariant functionals majorized by an invariant weight. .. 